\title{
Factores determinantes de la deserción escolar y expectativas de estudiantes que asisten a escuelas alternativas*
}

Óscar Germán Espinoza Díaz

https://orcid.org/oooo-0o01-7525-2980 Universidad de Tarapacá, Chile oespinoza@academia.cl

Javier Loyola Campos

https://orcid.org/oooo-0oo1-8336-6873 Programa Interdisciplinario de Investigaciones en Educación (PIIE), Chile javierloyolacampos@gmail.com
Luis Eduardo González Fiegehen

https://orcid.org/oooo-0003-1850-3899 Programa Interdisciplinario de Investigaciones en Educación (PIIE), Chile legonzalez.fiegehen@gmail.com

\section{Resumen}

Chile dispone de un sistema de "centros de segunda oportunidad" que ofrecen una oportunidad a desertores escolares entre 14 y 18 años de escuelas tradicionales para que completen sus estudios. Este artículo se propone un doble objetivo: por un lado, relacionar las características de los estudiantes de estos centros y sus familias con su historial de fracaso escolar (repitencia); por otro, establecer la relación existente entre las expectativas futuras declaradas por los estudiantes y un conjunto de variables de caracterización, incluido el historial de fracaso escolar. Para la recolección de información se aplicó una encuesta a 1.112 estudiantes, seleccionados de 18 de los 134 Centros de Segunda Oportunidad existentes en Chile. Se concluye que son relativamente pocas las variables personales y familiares vinculadas significativamente con el fracaso escolar y la repitencia. No existiría asi un conjunto fijo de rasgos que permita distinguir claramente a los estudiantes que solo han repetido una vez de aquellos que registran varias repitencias. Por otra parte, el análisis de las experiencias revela algunas importantes diferencias entre hombres y mujeres, en el sentido de que estas últimas parecen más preocupadas por su futuro, especificamente en lo relacionado con sus posibilidades laborales y educativas.

\section{Palabras clave (Fuente: tesauro de la Unesco)}

Deserción escolar; educación en Chile; educación media; pedagogías alternativas; rendimiento escolar.

* Artículo financiado por la Comisión Nacional de Investigación Científica y Tecnológica (Conicyt) a través del proyecto del Fondo Nacional de Desarrollo Científico y Tecnológico (Fondecyt) No. 1161433, titulado "Factores que inciden en el desarrollo de una oferta educativa para atender la continuidad educativa de niños, niñas y adolescentes que están fuera del sistema escolar formal y que son atendidos por las 'escuelas de segunda oportunidad'".

Recepción: 30/og/2020 | Envío a pares: 18/12/2020 | Aceptación por pares: 12/04/2021 | Aprobación: 17/04/2021

DOI: 10.5294/educ.2021.24.1.6

Para citar este artículo / To reference this article / Para citar este artigo

Espinoza, O., González, L. E., Loyola, J. (2021). Factores determinantes de la deserción escolar y expectativas de estudiantes que asisten a escuelas alternativas. Educación y Educadores, 24(1), 113-134. DOl: https://doi.org/10.5294/educ.2021.24.1.6 


\title{
Determining Factors of School Dropout and Expectations of Students Attending Alternative Schools
}

\begin{abstract}
Chile has a Second Chance Centers system that offers traditional school dropouts between 14 and 18 years old the opportunity to complete their studies. This article has a twofold objective: on the one hand, to relate the characteristics of the students of these centers and their families with their history of school failure (grade repetition); on the other, to establish the relationship between the students'future expectations and a set of characterizing variables, including the history of school failure. For data collection, we surveyed 1112 students selected from 18 of the 134 Second Chance Centers in Chile. We concluded that relatively few personal and family variables are significantly linked to school failure and grade repetition. Thus, there would not be a fixed set of traits to clearly distinguish students who have repeated a grade once from those who report several grade repetitions. Moreover, the analysis of the experiences reveals some significant differences between men and women in that the latter seem more concerned about their future, specifically their employment and educational possibilities.
\end{abstract}

\section{Keywords (Source: Unesco Thesaurus)}

School dropout; education in Chile; middle education; alternative education; school performance. 


\section{Fatores determinantes da desistência escolar e expectativas de estudantes de escolas alternativas}

\section{Resumo}

O Chile dispõe de um sistema de "centros de segunda oportunidade", os quais oferecem oportunidade a desistentes escolares entre 14 e 18 anos, de escolas tradicionais, para que terminem seus estudos. Neste artigo, é proposto um duplo objetivo: por um lado, relacionar as características dos estudos estudantes desses centros e suas familias com seu histórico de fracasso escolar (repetência); por outro, estabelecer a relação existente entre as expectativas declaradas pelos estudantes e um conjunto de variáveis de caracterização, incluído o histórico de fracasso escolar. Para a coleta de dados, foi aplicada uma enquete a 1.112 estudantes, selecionados de 18 dos 134 centros de segunda oportunidade no Chile. Conclui-se que são relativamente poucas as variáveis pessoais e familiares vinculadas significativamente com o fracasso escolar e com a repetência. Não existe assim um conjunto fixo de traços que permita diferenciar claramente os estudantes que somente repetiram uma vez daqueles que registram várias repetências. Por sua vez, a análise das experiências revela algumas importantes diferenças entre homens e mulheres, no sentido de que estas últimas parecem mais preocupadas com seu futuro, especificamente no que se refere às possibilidades profissionais e educacionais.

\section{Palavras-chave (Fonte: tesauro da Unesco)}

Deserção escolar; educação no Chile; ensino médio; pedagogias alternativas; desempenho escolar. 
En Chile, las tasas de finalización de la escuela secundaria son relativamente altas: un $88 \%$ se encuentra en el grupo etario entre 18 y 25 años (OECD, 2018). No obstante lo anterior, es motivo de gran preocupación que quienes no llegan a completar este ciclo pertenezcan, casi en su totalidad, a los estratos socioeconómicos más bajos (Biblioteca del Congreso Nacional, 2014). Desde 1989 en este país algunas organizaciones no gubernamentales comenzaron a establecer escuelas de segunda oportunidad dirigidas a desertores escolares (Alvarado et al., 2014). Ahora bien, no fue sino hasta tiempos más bien recientes cuando el gobierno incluyó a adolescentes fuera de la escuela (menores de 18 años) en programas de preparación de adultos para el empleo. Más allá de estos esfuerzos, aquellos que se beneficiaron de dichos programas fueron solo una parte de esta población, con más del $50 \%$ de los ingresantes sin haber logrado concluir el año escolar (Espinoza et al., 2018, 2020a; MIDE-UC y Sename, 2016; Mineduc, 2015).

Concretamente, la Coordinación Nacional de Educación de Personas Jóvenes y Adultas (EPJA) -dependiente del Ministerio de Educación (Mineduc)ofrece tres modalidades, complementarias a la típica, orientadas a todos aquellos que no han completado los 12 años de escolaridad obligatoria que contempla la legislación chilena. En primer lugar, se encuentra la modalidad "regular", implementada por los Centros de Educación Integrada de Adultos (CEIA) y las "terceras jornadas", a cargo de escuelas que tienen una tercera jornada vespertina orientada a la formación de adultos. Luego está la modalidad "flexible", que atiende las necesidades específicas de la población trabajadora y activa en el mercado laboral. Finalmente, la tercera modalidad corresponde a los llamados "proyectos de reinserción escolar", orientados a niños y adolescentes de entre 8 y 18 años de edad desescolarizados, que poseen antecedentes de fracaso escolar reiterado y/o están en situación de vulnerabilidad socioeducativa; en otras palabras, que están fuera del sistema regular de enseñanza o bien presentan graves dificultades para continuar sus estudios (Mineduc, 2015). De este modo, lo más característico de esta modalidad en comparación con las dos anteriores se vincula con el hecho de que se focaliza en menores de edad y atiende a estudiantes que están fuera de la escuela y en alto riesgo. Es por esta razón que, por sus características de focalización, y pese a que esta oferta educativa está alojada en la EPJA, los proyectos de reinserción pueden ser comprendidos como la única iniciativa pública de escuela de segunda oportunidad (Espinoza et al., 2018, 2019a; Pizarro, De la Vega y Ormazábal, 2016; Vivanco, 2014).

Precisamente, con el propósito de atraer y atender de mejor forma las necesidades de los desertores escolares adolescentes, en el 2015 el Mineduc estableció un sistema de Centros de Segunda Oportunidad. Esta denominación le fue otorgada a las instituciones ejecutoras que implementan programas de reinserción educativa (tercera modalidad) focalizadas en estudiantes de entre 14 a 18 años que no asisten a la escuela convencional (Escobar, Berlien y Ostoic, 2016; Espinoza et al., 2016; Mineduc, 2015; Romo y Cumsille, 2020). Se trata de programas intensivos donde el progreso académico es acelerado: en un año calendario los estudiantes pueden completar dos grados de educación secundaria (CIAE, 2019; Espinoza et al., 2018, 2019a).

Los Centros de Segunda Oportunidad se han mostrado ineficaces a la hora de retener a sus estudiantes (Mineduc, 2010, 2015). Esta situación invita a preguntarse respecto del tipo de proceso educativo que resulta pertinente para la población menor de edad que se encuentra fuera del sistema escolar convencional desde hace dos años o más. Sobre los programas de reescolarización, se ha señalado que existiría cierta tensión entre esta función y su labor de retención. Existen muchas causas probables de esta fricción, entre ellas la falta de recursos económicos y humanos y/o personal capacitado que permita hacerse cargo de ambas funciones al mismo tiempo y de forma adecuada (Alvarado et al., 2014; Brusilovsky y Cabrera, 2005; Brusilovsky, Cabrera y 
Kloberdanz, 2010; McDermott, Anderson y Zaff, 2017; Schuller y Watson, 2009; Vallee, 2017). Asimismo, esta tensión o sobrecarga podría desembocar en un quehacer pedagógico debilitado en la escuela de segunda oportunidad (Ceaal, 2013). Justamente se ha puesto un gran énfasis en las capacidades pedagógicas y didácticas de estos centros, las cuales debiesen ser más flexibles y adaptables en comparación con las de la escuela regular, esto en virtud de las características de la población a la que atienden. Se ha destacado entonces la necesidad de fortalecer y potenciar dichas capacidades en los centros de reescolarización para así ofrecer experiencias educativas más relevantes y enriquecedoras a sus estudiantes, que contribuyan a su permanencia y eventual culminación de su educación escolar (Day et al., 2013; Espinoza et al., 2014; Osorio, 2013).

En Chile, si bien en los últimos años se han desarrollado una serie de estudios focalizados en los procesos de reescolarización que tienen lugar en las escuelas de segunda oportunidad (Espinoza et al., 2016, 2020b; Romo y Cumsille, 2020), se carece todavía de información suficiente que permita conocer y comprender cabalmente el modo en que los programas de reinserción están funcionando. Un factor crítico aquí es la adecuación que debe existir entre la oferta de los programas y las características de la población estudiantil que concurre a ellos. En la actualidad hacen falta nuevos estudios que permitan caracterizar a los estudiantes de estas escuelas, con el objetivo de establecer sus rasgos distintivos y relevar los factores determinantes que pueden ayudar a retener a estos adolescentes y jóvenes, y así evitar otro fracaso escolar (Espinoza et al., 2019b).

El presente trabajo busca ser un aporte en este sentido, al plantearse como propósito central analizar ciertas características de los estudiantes de los Centros de Segunda Oportunidad y sus ambiciones y expectativas futuras. Específicamente, se busca: 1) relacionar las características de los estudiantes y sus familias con su historial de fracaso escolar (repitencia), en el entendido de que esto llevó al abandono temporal de sus estudios y su posterior ingreso al centro de segunda oportunidad; y 2) establecer la relación existente entre las expectativas futuras declaradas por los estudiantes (incluyendo la variable género) y un conjunto de variables de caracterización personal y familiar que inciden en el historial de fracaso escolar.

\section{Marcos interpretativos para comprender el fracaso y la deserción escolar}

Los "desertores escolares" en la actualidad -así como también quienes se encuentran en riesgo de abandonar de forma definitiva el sistema escolarson entendidos como aquellos niños, adolescentes y jóvenes que no se han matriculado en ninguna escuela (Lamb y Markussen, 2011; Unesco, 2012). Esta categoría incluye a quienes han decidido abandonar la escuela voluntariamente, al mismo tiempo que los estudiantes que han sido expulsados o forzados a desertar por motivos de fracaso escolar o conducta antisocial (Bradley y Renzulli, 2011; Ramírez, 2020). Entre los desertores escolares existen estudiantes con problemas de comportamiento -por ejemplo, conductas criminales-, discapacidad física, dificultades de carácter psicológico, limitaciones intelectuales, adicción a las drogas y/o paternidad temprana, entre otras muchas cuestiones (Fernández et al., 2016, 2011; Hawkins, Jaccard y Needle, 2013). Quienes abandonan la escuela tradicional son precisamente los potenciales candidatos para integrarse a las llamadas "escuelas de segunda oportunidad" o escuelas alternativas. Independientemente de las razones, estas escuelas han sido creadas justamente con la finalidad de reintegrar a quienes no lograron finalizar su proceso formativo en el sistema educativo formal (Day et al., 2013; McDermott et al., 2017; Porowski, O'Conner y Luo, 2014). La comprensión del por qué los estudiantes abandonan los programas de reinserción o reescolarización -que afecta mayormente a la población más joven que asiste a ellos- se puede abordar de manera similar a otros procesos parecidos de deserción en otros niveles del 
sistema escolar tradicional. Así, los enfoques que se han utilizado para la comprensión de estos últimos procesos pueden arrojar luces sobre las causas que están detrás del abandono en las escuelas de segunda oportunidad (Espinoza et al., 2018).

El abandono de los estudios puede ser de carácter temporal o definitivo, siendo el primero antesala del segundo. La deserción definitiva se ha entendido como el proceso de alejamiento y abandono, voluntario o forzoso, de la escuela, en cuanto espacio cotidiano, esto por circunstancias internas y/o externas al estudiante (D’Alessandre y Mattioli, 2015; Sabates et al., 2010).

Son varias las perspectivas desde las cuales se ha abordado el fenómeno de la deserción escolar. Una de ellas sostiene que el fracaso escolar estaría asociado con la repitencia y el rezago (Adelman y Székely, 2017; González, Espinoza y López, 2013; Noltemeyer, Ward y Mcloughlin, 2015). De manera general, los gatillantes del abandono escolar usualmente se han agrupado en dos grandes categorías. Por una parte, variables extraescolares, tales como la pobreza y la vulnerabilidad (Peña y Toledo, 2017), la situación socioeconómica (De Witte y Rogge, 2013; Ingrum, 2007; Rumberger y Lim, 2008), la búsqueda de trabajo, el origen étnico, la desintegración familiar y las limitadas expectativas de la familia con respecto a la educación (Castillo, 2003; Espinoza et al., 2014 2016, 2020a; Foley, Gallipoli y Green, 2014; Hawkins et al., 2013). Por ejemplo, algunas investigaciones han puesto en evidencia cómo la deserción escolar se vincula con razones económicas, dado el vínculo entre el abandono de la escuela y la necesidad de ingresar tempranamente al mundo laboral para satisfacer requerimientos familiares (Perreira, Harris y Lee, 2006). Por otra parte, se ha establecido que el clima educacional generado por la familia y la propia escuela se encuentra estrechamente vinculado con la probabilidad de permanecer en la escuela (Antelm, Gil y Cacheiro, 2015; Espinoza et al., 2016; López, Carvajal y Urrea, et al., 2013; Peña, Soto y Calderón, 2016; Salinas et al., 2017). La segunda categoría corresponde a la de los factores intraescolares que, como detonantes del fracaso y el abandono temprano, incluyen variables tales como el autoritarismo docente, los problemas de comportamiento, el bajo rendimiento académico y el adultocentrismo (Darling y Cook, 2018; Espinoza et al., 2014; Hennig, Pineda y Vargas, 2019; Rumberger y Lim, 2008). Algunos estudios han sostenido que las condiciones que gatillan el fracaso escolar son creadas por los mismos establecimientos educativos. En este sentido, el abandono escolar no se vincula únicamente con una pérdida de interés de parte de los estudiantes, puesto que son las propias escuelas, de una u otra manera, los terminan expulsando al estudiante (Glewwe et al., 2011). En esta misma línea, se ha destacado que las escuelas no cuentan con estrategias pedagógicas adecuadas para atender a estudiantes en situación de extrema pobreza (Amitay y Rahav, 2018; Tedesco, 1998). Es justamente en los establecimientos educativos vinculados a los sectores más vulnerables donde son notoriamente más frecuentes las situaciones de repitencia, expulsiones y sobreedad del alumnado, en cuanto antesalas de la deserción definitiva (Noltemeyer et al., 2015). Ahora bien, más allá de esta división entre factores extra e intraescolares, lo cierto es que la deserción escolar es un fenómeno complejo vinculado con variables de diversa índole y, por lo tanto, no debe considerarse como la consecuencia de un suceso aislado (Bradley y Renzulli, 2011; D'Alessandre y Mattioli, 2015; Lamb y Markussen, 2011; Rumberger, 2011).

En cuanto a la población que concurre a escuelas de segunda oportunidad, la mayor parte cuenta con experiencias escolares marcadas por el fracaso escolar -repitencia, ausentismo y bajo rendimiento-, así como por condiciones de carácter psicosocial, que se expresan en problemas de diversa índole (Ceaal, 2013; Espinoza et al., 2019a; Salva, Nadal y Melià, 2016). La probabilidad de caer en dinámicas excluyentes y socialmente desintegradoras -como son la cesantía (desempleo), la criminalidad y/o la drogadicción-son mayores en quienes han abandonado la escuela convencional (Estrada, 2015). 


\section{Expectativas futuras en la adolescencia}

La adolescencia se suele entender como una etapa de transición entre la niñez y la adultez y, como tal, constituye un período de cambio y ajuste, fundamental en la construcción de un proyecto vital significativo y diferenciado (Crone y Dahl, 2012; Behtoui, 2017). Constituye así una fase crucial en la que el futuro adquiere gran importancia, estableciéndose metas personales y expectativas futuras que orientarán el tránsito hacia la vida adulta (Laghi et al., 2011; Mills y McGregor, 2014).

Concretamente, las expectativas de futuro se refieren al grado en que los individuos esperan que ocurra un evento, lo que a su vez influye en la planificación y establecimiento de metas que luego guiarán la conducta y la toma de decisiones (Seginer, 2009). Dichas proyecciones no solo son el resultado de un proceso cognitivo individual, sino que también están condicionadas por los contextos socioculturales en que los individuos se encuentran insertos. En este sentido, la mirada de futuro de los adolescentes se puede ver influida por la familia, el grupo de pares, la institución educativa, el nivel socioeconómico y las normas y valores, entre otras cuestiones (León y Sugimaru, 2017; Palomar y Victorio, 2017; Seginer, 2009; Verdugo, 2017).

Las expectativas futuras en la adolescencia se pueden relacionar con una serie de dominios, siendo los más comunes las preocupaciones vinculadas con la educación, el trabajo, la familia/matrimonio y las preocupaciones personales, que incluyen las posesiones materiales (Bandura, 2006). De estas, particular atención han recibido las metas y expectativas de los adolescentes referidas al futuro educacional y laboral (Palomar y Victorio, 2017). Dada la estrecha relación entre educación y ocupación laboral, las expectativas educacionales y laborales también están relacionadas. Las primeras están entrelazadas con el futuro económico de los jóvenes, pues la expectativa de conseguir mayores niveles educativos se vincula con la expectativa de acceder a mejores empleos y con ello lograr mayores retribuciones económicas. Así, la esperanza de alcanzar metas de educación superior impulsa su decisión de conseguirlas, y estos niveles académicos más altos a su vez posibilitan mayores ingresos económicos, ayudando a la movilidad social ascendente (Palomar y Victorio, 2016).

Las expectativas educacionales constituyen, de este modo, predictores decisivos de las elecciones que realizan los adolescentes (por ejemplo, continuar o abandonar sus estudios escolares, ingresar a la universidad), estando fuertemente vinculadas con el nivel educativo y el logro laboral alcanzados posteriormente (Parker et al., 2016). La formación de expectativas específicas -educacionales, laborales, etc.- depende de varios factores. Por ejemplo, se ha asociado un mayor nivel socioeconómico con un nivel de expectativas más alto (Gutman, Schoon y Sabates, 2012). En este sentido, el residir en barrios con altos índices de pobreza reduciría la exposición de los adolescentes a modelos ejemplares de empleos bien remunerados y de oportunidades laborales que puedan alimentar sus expectativas y, de este modo, el establecimiento de metas educacionales y laborales futuras con propósitos de movilidad social (Donlan, Prescott y Zaff, 2016).

Asimismo, las expectativas de los padres con respecto a la educación de sus hijos son un aspecto importante en la explicación de las expectativas educacionales y laborales de los adolescentes (OECD, 2017; Palomar y Victorio, 2016). Las altas expectativas de los padres habitualmente implican mensajes, actitudes y acciones que comunican el valor de la educación; de no existir este apoyo y orientación en la familia, los adolescentes presentarían mayores dificultades para definir objetivos educativos y percibirían mayores barreras para alcanzar sus metas (Ceja, 2004; Maxwell, McNeely y Carboni, 2016).

Junto con el nivel socioeconómico y las expectativas parentales, también se ha destacado el rol de un buen rendimiento escolar en la definición de expectativas laborales y educacionales más altas 
(Gutman et al., 2012; OECD, 2017; Tynkkynen, Vuori y Salmela, 2012). Por el contrario, una historia académica caracterizada por el fracaso escolar o la repitencia, así como por los problemas conductuales y de aprendizaje, merma las expectativas educacionales de los adolescentes (Jacob y Wilder 2010; Stinebrickner y Stinebrickner, 2013). Respecto de la expectativa de cursar estudios universitarios, se ha dicho que ella declina cuanto más bajo sea el rendimiento académico -lo mismo frente a la escasez de recursos económicos y el poco apoyo del entorno familiar-, con lo que los adolescentes tenderían a eliminar la aspiración universitaria de su horizonte de posibilidades (Bozick et al., 2010; Parker et al., 2016). De este modo, en el caso de los estudiantes de los Centros de Segunda Oportunidad, caracterizados por un historial de fracaso escolar previo al abandono de la escuela regular, se esperaría que la expectativa universitaria no fuera recurrente en ellos.

Otros factores que han sido destacados como predictores de las expectativas son el sentido de autoeficacia o la confianza en las propias habilidades (Stewart et al., 2007), el apoyo brindado por la escuela mediante los docentes y compañeros (Reddick et al., 2011), la etnia (Bohon, Kirkpatrick y Gorman, 2006) y el género (Alarcón, Parella y Yiu, 2014).

\section{Metodología}

El presente estudio es de carácter fundamentalmente descriptivo, con un componente relacional, ya que se propone caracterizar a los estudiantes y sus familias buscando las relaciones, directas e indirectas, con el historial de fracaso escolar y las expectativas futuras de los estudiantes -de diverso tipo, entre ellas educacionales y laborales- de los Centros de Segunda Oportunidad. El diseño de investigación utilizado corresponde a uno no experimental, basado en un análisis ex-post facto. Este fue adoptado por la imposibilidad de manipular las variables que intervinieron en el proceso de reescolarización.
En este estudio se entiende por Centros de Segunda Oportunidad las experiencias educativas que atienden a niños, adolescentes y jóvenes de 18 años o menos. Ahora bien, considerando que los Centros de Educación Integrada de Adultos (CEIA), dentro de la modalidad "regular", tienen una cobertura nacional y concentran el mayor número de estudiantes, se decidió analizar la situación de sus estudiantes de hasta 18 años de edad. Y es que en la actualidad poco más del 50\% de la población que asiste a la oferta de Educación de Personas Jóvenes y Adultas (EPJA) está constituida por adolescentes en este rango de edad; es decir, se observa un proceso de "adolescentización" de una educación que tradicionalmente se pensó para alfabetizar a población adulta (Espinoza et al., 2019a).

Los participantes del estudio fueron seleccionados utilizando muestreo por conglomerados, pues existen estudios (Mineduc, 2010) que muestran que los desertores se distribuyen proporcionalmente a través de las regiones de Chile, en agrupaciones naturales (conglomerados) correspondientes a la ubicación de los CEIA. El marco muestral fue construido utilizando datos estadísticos de 2016, con la asistencia técnica del Mineduc. El universo incluyó 30.262 adolescentes entre los 13 y los 18 años de edad, matriculados en los CEIA de todo el país. Como modo de obtener una muestra total con un nivel de confianza de al menos 95\%, primeramente se seleccionó un determinado número de centros en cada región en relación con la matrícula regional total de los CEIA. Esto derivó en la selección de un total de 18 centros sobre un total de 134 .

Durante los meses de noviembre y diciembre de 2016 fue visitado cada uno de estos centros. Los estudiantes fueron seleccionados de manera probabilística sobre la base de la matrícula total de su centro, resultando así un tamaño muestral de 1.112 estudiantes a nivel nacional con un error muestral de 2,88\%. Los datos de esta investigación fueron obtenidos a través de un cuestionario administrado 
a los estudiantes en los centros educativos, el cual incluyó, entre otras cosas, preguntas acerca de sus familias y condiciones de vida, características personales y comportamientos habituales, ambiciones para el futuro, y participación y evaluación de las actividades de los Centros de Segunda Oportunidad.

Los resultados se presentan en dos secciones. La primera describe a los estudiantes y sus familias, con énfasis en la relación entre repitencia y una serie de factores. La segunda sección, por su parte, indaga en las expectativas futuras de los estudiantes y cómo estas varían en función de ciertas variables incluidas en la encuesta. En concordancia con lo anterior y, por ende, con el objetivo central de la investigación, el análisis de los datos se lleva a cabo fundamentalmente mediante análisis estadístico descriptivo (frecuencias y porcentajes), tablas de contingencia, coeficientes de correlación y análisis de componentes principales.

\section{Resultados}

\section{Caracteristicas generales de los estudiantes y sus familias}

\section{Sexo, edad e historial de repitencia:}

La muestra está compuesta por 498 mujeres $(44,8 \%)$ y 614 hombres $(55,2 \%)$, los cuales se encuentran distribuidos de forma relativamente equitativa en los distintos establecimientos. Más de la mitad de los estudiantes de la muestra tienen 17 o 18 años de edad. Hombres y mujeres no difieren en su distribución de edades. Los 18 centros varían ampliamente en la distribución etaria de sus estudiantes: en algunos la mitad tiene de 13 a 15, mientras que en otros la mayoría tiene 17018 años. Los estudiantes se diferencian entre sí en cuanto al número de ocasiones en que han repetido de grado en la escuela tradicional antes de ingresar a sus respectivos Centros de Segunda Oportunidad. Un grupo de estudiantes no ha repetido nunca (11,4\% de la muestra), mientras que más de un tercio $(34,4 \%)$ ha repetido dos veces y el 10,1\% lo ha hecho cuatro o más veces.
La encuesta no preguntó en qué momento de su trayectoria escolar los estudiantes repitieron. Ante la ausencia de esta información específica, se estimó cuándo estos adolescentes experimentaron por primera vez el fracaso escolar, es decir, cuándo fue la primera oportunidad en que repitieron de grado en el sistema escolar formal. En primer lugar, la edad se agrupó en tramos de dos años para aproximarse a la distribución real de las edades de los estudiantes en los distintos grados. Luego, se asumió que los estudiantes repitieron al año o grado siguiente del que les correspondería según su tramo de edad. Sobre esta base se estimó, para cada estudiante, el grado repetido por primera vez y su edad en ese momento (Tabla 1).

Tabla 1. Edad aproximada y grado estimado al momento de la primera repitencia en la escuela tradicional

\begin{tabular}{|c|c|c|c|}
\hline $\begin{array}{c}\text { Edad } \\
\text { aproximada }\end{array}$ & $\begin{array}{c}\text { Grado } \\
\text { estimado* }\end{array}$ & $\mathbf{N}$ & \% \\
\hline $8-9$ & 3 & 2 & 0,2 \\
\hline $9-10$ & 4 & 5 & 0,4 \\
\hline $10-11$ & 5 & 22 & 2,0 \\
\hline $11-12$ & 6 & 39 & 3,5 \\
\hline $12-13$ & 7 & 98 & 8,8 \\
\hline $13-14$ & 8 & 204 & 18,3 \\
\hline $14-15$ & 9 & 246 & 22,1 \\
\hline $15-16$ & 10 & 298 & 26,8 \\
\hline $16-17$ & 11 & 142 & 12,8 \\
\hline $17-18$ & 12 & 56 & 5,0 \\
\hline & & 1112 & 100 \\
\hline
\end{tabular}

* Tercero a octavo corresponden a grados del ciclo primario o básico, mientras que el tramo 9-12 es el ciclo secundario o medio.

Fuente: elaboración propia.

Esta estimación indica que la mayoría de los estudiantes que componen la muestra no comenzaron a repetir antes de alcanzar la edad de 13 años. En el sistema chileno, esta edad corresponde aproximadamente al octavo año básico, vale decir, al último año del segundo ciclo de la educación primaria. 
De acuerdo con estas estimaciones, un tercio de los estudiantes matriculados en las escuelas alternativas repitieron en el segundo ciclo de la educación básica convencional, esto es, antes de ingresar al primer año de la educación secundaria. Los otros dos tercios comenzaron a repetir una vez que ingresaron al ciclo secundario de la educación tradicional.

Las mujeres presentan menores probabilidades que los hombres de haber repetido por primera vez en octavo básico. La probabilidad de fracaso escolar aumenta en ambos grupos desde la edad de 13, pero más rápidamente en el caso de los hombres. Las mujeres tienen más probabilidades que los hombres de repetir por primera vez solo cuando han alcanzado los 15 años de edad. En esta muestra, el $16,3 \%$ de las mujeres nunca ha repetido, comparado con el 7,5\% de los hombres. La mayoría de los estudiantes (89\%) ha repetido uno o más grados, con los hombres repitiendo más veces $(2,1)$ que las mujeres $(1,8)$. Aunque pequeñas, todas estas diferencias son estadísticamente significativas.

\section{Caracteristicas familiares:}

La mayoría de los estudiantes de la muestra $(84,6 \%)$ viven con uno o con ambos padres (y con abuelos y hermanos). La Tabla 2 muestra cómo estas proporciones varían por nivel educacional de los padres o tutores. Alrededor del 34\% vive en un hogar con ambos padres, otro $42 \%$ vive solo con su madre y el $10 \%$ vive solo con su padre. Mujeres y hombres viven en condiciones de vivienda similares. La edad o grado en la que el estudiante repitió por primera vez no tiene relación con la persona con la que convive el estudiante. Menos del $20 \%$ de los padres o tutores ha recibido algún tipo de educación postsecundaria. Hay una pequeña pero significativa diferencia en el nivel educacional de los diferentes tipos de padres o tutores. Los niveles educativos son más altos cuando ambos padres están presentes, en hogares con presencia solo de la madre, y cuando un tío o tía es el tutor/a.

Aproximadamente el $56 \%$ de los encuestados describieron la situación económica de su hogar como regular, mala o muy mala. Esta situación es más severa en hogares encabezados solo por la madre, un poco menos grave en los hogares con presencia de ambos padres, y mejor en los hogares encabezados por el padre, los abuelos o la tía o tío ( $p<$ 0,005 ). La situación económica del hogar se vincula con el tipo de relación existente entre los estudiantes y sus padres o tutores. Estas relaciones son peores en los hogares con más problemas económicos (Chi cuadrado $=227,32, \mathrm{p}<0$ ). Por otra parte, ninguna

Tabla 2. Nivel educacional alcanzado por los padres o tutores (\%)

\begin{tabular}{|c|c|c|c|c|c|c|c|}
\hline $\begin{array}{c}\text { Nivel } \\
\text { educativo }\end{array}$ & $\begin{array}{c}\text { Ambos } \\
\text { padres }\end{array}$ & Solo madre & Solo padre & Abuelos & Tío o tía & Hermano & Total \\
\hline Ninguno & 1,3 & 1,9 & 1,9 & 2,3 & 0 & 13,6 & $2,2(24)$ \\
\hline Básico & 32,9 & 31 & 33 & 34,5 & 44,1 & 34,1 & $32,6(363)$ \\
\hline Secundaria & 46,8 & 48,8 & 51,9 & 52,9 & 35,3 & 43,2 & $48,1(535)$ \\
\hline Técnico & 10,7 & 10,1 & 6,6 & 5,7 & 14,7 & 4,5 & $9,5(106)$ \\
\hline Superior & 8,3 & 8,1 & 6,6 & 4,6 & 5,9 & 4,5 & $7,6(84)$ \\
\hline N & 374 & 467 & 106 & 87 & 34 & 33 & 1.112 \\
\hline$\%$ & 33,6 & 42 & 9,5 & 7,8 & 3,1 & 4 & 100,0 \\
\hline
\end{tabular}

${ }^{*}$ Chi cuadrado $=40,375, p=0,004$.

Fuente: elaboración propia. 
de estas variables se asocia con la edad de los estudiantes cuando repitieron por primera vez ni con el número de veces que repitieron.

En cuanto a otro tipo de problemas, se les pidió señalar a los estudiantes, de una lista de nueve situaciones problemáticas, aquellas que han ocurrido en sus familias. Las alternativas incluyeron falta de comunicación, problemas con el alcohol y abuso físico o psicológico. La mayoría de los encuestados reportaron pocas situaciones: el 20\% no mencionó ninguna y el $34 \%$ solo una. La media de situaciones mencionadas fue de 1,92. Dos opciones se relacionaron significativamente con la frecuencia de la repitencia. Una de ellas es el uso de drogas, que fue más citada por los hombres (15\%) que por las mujeres (8\%). La otra corresponde a las dificultades con los hermanos, que fue mencionada en mayor medida por las mujeres (19,3\%) que por los hombres (12,1\%) El número de opciones mencionadas se correlaciona con las respuestas a la evaluación de las relaciones con los padres y tutores.

El análisis de componentes principales arrojó la existencia de dos grupos de estudiantes: aquellos sin un patrón definido de elecciones (782) y aquellos que mencionaron falta de comunicación, problemas económicos, malas relaciones entre padres e hijos y falta de tiempo para compartir juntos (330). Hay una tendencia en los hombres (73,3\% comparada con el $66,7 \%$ de las mujeres) a posicionarse en la categoría de patrón no definido de situaciones familiares, mientras que las mujeres más a menudo se encuentran en el conglomerado de falta de comunicación $(33,3 \%$ versus $26,7 \%)$. No hay diferencias entre los dos conglomerados en términos del número promedio de veces que repitieron.

Las familias y hogares de los estudiantes varían en una serie de dimensiones, tales como: la estabilidad marital, el nivel de ingresos familiares y la escolaridad de los padres, cuestiones comúnmente asociadas con el fracaso escolar y la repitencia. No se encuentra, sin embargo, ninguna relación signifi- cativa entre estas variables. Puede ser que los estudiantes que asisten a Centros de Segunda Oportunidad no sean representativos del grupo más amplio de aquellos que han repetido. No se puede someter a prueba esta hipótesis con los datos disponibles, pero se pueden buscar otras variables que den cuenta de por qué algunos estudiantes experimentan el fracaso escolar más tempranamente que otros.

La Tabla 3 muestra que los estudiantes presentan mayores probabilidades de repetir en el ciclo primario, si no se encuentran viviendo con ambos padres: el $73 \%$ de los repitentes de educación primaria se encontraban en esta situación, comparado con el 63,15\% de aquellos que repitieron en el ciclo secundario. Por otra parte, los estudiantes cuyos padres tienen un nivel educativo menor presentan mayores probabilidades de repetir en el ciclo secundario. Alrededor del $60 \%$ de los estudiantes repitentes en el ciclo primario son hombres, comparado con el $52 \%$ registrado del ciclo secundario.

Tabla 3. Proporción de estudiantes repitentes en el ciclo primario comparado con aquellos repitentes en el ciclo secundario, por características

\begin{tabular}{|c|c|c|c|}
\hline & $\begin{array}{c}\text { Ciclo } \\
\text { primario }\end{array}$ & $\begin{array}{c}\text { Ciclo } \\
\text { secundario }\end{array}$ & $\begin{array}{c}\text { Nivel de } \\
\text { significación }\end{array}$ \\
\hline $\begin{array}{c}\text { Familia no } \\
\text { intacta }\end{array}$ & $73,0 \%$ & $63,1 \%$ & 0,001 \\
\hline $\begin{array}{c}\text { Bajo nivel } \\
\text { educacional } \\
\text { de los padres }\end{array}$ & 31,4 & 36,5 & 0,050 \\
\hline Hombre & 60,8 & 52,4 & 0,005 \\
\hline
\end{tabular}

Fuente: elaboración propia.

\section{Expectativas de los estudiantes}

\section{Ambiciones futuras:}

A los estudiantes se les presentaron 10 alternativas a la pregunta respecto de qué quieren hacer en el futuro. Las mujeres mencionan, en promedio, 2,4 de las opciones listadas en el cuestionario, mientras que los hombres señalan 2,O. Alrededor del $28 \%$ de 
los estudiantes no señala ninguna de las alternativas listadas, el 36\% señala solo una, el 16\% dos y el $11 \%$ tres. Ningún encuestado señala más de cinco. El deseo más popular para el futuro es el de conseguir un buen trabajo (43\%), seguido de cerca por ganar más dinero (41\%). Una proporción considerable (30\%) señaló que tan solo le gustaría estar con sus amigos. Por otra parte, al 23\% le gustaría terminar la escuela, al 18\% le gustaría estudiar en la universidad y al $17 \%$ le gustaría obtener un grado técnico (postsecundario) o profesional. Únicamente el 14\% declaró que le gustaría formar una familia.

Las mujeres que han repetido más frecuentemente presentan mayores probabilidades de haber pensado sobre diferentes opciones para su futuro que aquellas con menos repitencias, pero la diferencia no es amplia. Para los hombres, por su parte, los planes de futuro no están vinculados con cuántas veces han repetido. En general, no hay relación entre frecuencia de repitencia y elección de futuras actividades.

Ahora bien, la cantidad de diferentes opciones para el futuro en las que han pensado los estudiantes están vinculadas con sus experiencias en el hogar. Los estudiantes que declaran que sus relaciones en el hogar son "muy buenas" eligen en promedio una opción para el futuro, pero una media de dos, si perciben estas relaciones como "muy malas" ( $x 2$ = 31,38; $\mathrm{p}<0,000)$. Por su parte, los estudiantes que ven la situación económica familiar como "muy mala" mencionan más opciones para el futuro $(2,4$ en promedio) que quienes la perciben como "muy buena" (o,86 opciones).

La Tabla 4 reporta estas relaciones habiendo combinado las variables en cuatro conjuntos de opciones futuras, referidas a dinero o empleo, educación, dejar la casa (formar familia, mudarse, poseer casa propia), y otros (hacer nada, solo estar con los amigos, realizarse como persona). El número promedio de opciones mencionadas en cada conjunto es significativo, pero moderado y negativamente correlacionado con la evaluación que hacen los estudiantes de sus relaciones con otros en su hogar o la situación financiera familiar. En otras palabras, los estudiantes que evalúan las relaciones en el hogar y la situación financiera familiar como "muy buenas" mencionan menos opciones para el futuro.

\section{Razones posibles para dejar de estudiar:}

En el cuestionario se les preguntó a los estudiantes por las razones que podrían tener para dejar su centro. Alrededor de la mitad de la muestra estuvo de acuerdo en que no tienen razón alguna para dejarlo en este momento. Entre quienes ofrecieron explicaciones posibles, los hombres tienen más probabilidades de decir que podrían dejar el centro por ser expulsados (23\% comparado con el $15 \%$ de las mujeres); porque les gustaría trabajar ( $21 \%$ versus $16 \%)$ o por no querer seguir estudiando $(11 \%$

Tabla 4. Correlación entre relaciones en el hogar y situación financiera familiar y número de diferentes opciones para el futuro

\begin{tabular}{|c|c|c|c|c|c|}
\hline \multirow{2}{*}{} & \multicolumn{5}{|c|}{ Categorias de opciones para el futuro } \\
\cline { 2 - 6 } & Dinero/trabajo & Educación & Dejar la casa & Otros & No. de opciones elegidas \\
\hline Relaciones en el hogar & & & & & \\
\hline Situación financiera familiar & 2,76 & 3,18 & 3,23 & 56,02 & 0 \\
\hline N & 649 & 185 & 278 & & \\
\hline
\end{tabular}

* Todas las correlaciones son significativas a 0,01.

Fuente: elaboración propia. 
versus $5 \%$ ). Las mujeres son más propensas a decir que sería por problemas familiares (21\% versus $12 \%)$ o por problemas con compañeros de clase $(11 \%$ versus $7 \%$ ). Mientras más a menudo una mujer ha repetido un curso, más probabilidad existe de que considere la expulsión como una razón para dejar el curso actual y menos probabilidad de que piense que los problemas familiares puedan ser una causa para el abandono.

\section{Valoración de la educación:}

Los estudiantes debieron elegir las alternativas que mejor representan su sentir entre un total de seis opiniones acerca del valor de la educación para su futuro. Tres de estas posibles opiniones minimizan su importancia. La respuesta más común fue positiva (66\%): "Es lo único que me permitirá salir adelante". El análisis de componentes principales (Tabla 5) arroja tres componentes diferenciados. Los hombres prefieren las respuestas más generales (que la educación haría posible surgir en la vida), mientras que las mujeres prefieren identificar lo que pasaría, por ejemplo, de recibir un título postsecundario. El más grande de los tres conglomerados, con una respuesta positiva al ítem anteriormente mencionado, incluyó a 572 estudiantes. El segundo más amplio, con 421, tiene en su centro el ítem "me servirá para ser profesional o técnico". Un pequeño grupo de 66 personas no se mostró impresionado por su centro educativo ni por la educación recibida. Mencionan "para mí no es tan importante". Como con otras preguntas, los hombres se ubicaron en el conglomerado menos específico, mientras que las mujeres más frecuentemente señalaron la obtención de un grado académico como su objetivo. Las diferencias son pequeñas, pero estadísticamente significativas ( $p=0,011)$. Independientemente del sexo, los estudiantes en el conglomerado orientado al grado académico han repetido en promedio menos veces que aquellos que se ubican en el conglomerado menos específico.

\section{Discusión}

La revisión de los datos muestra que, tal como también lo han revelado estudios en otros países (Adelman y Székely, 2017; Bowers, 2010; Bowers, Sprott y Taff, 2013; De Witte y Rogge, 2013; Román, 2013; Rumberger, 2011), no existe un factor único o un conjunto reducido de variables que permitan explicar por qué ciertos estudiantes presentan dificultades

Tabla 5. Análisis de componentes principales de las respuestas a la pregunta "¿Qué opinas sobre la importancia de la educación que estás recibiendo para tu futuro?"

\begin{tabular}{|c|c|c|c|c|c|c|}
\hline \multirow{2}{*}{ Alternativa } & \multicolumn{3}{|c|}{ Componente } & \multirow{2}{*}{ Mujeres \% } & \multirow{2}{*}{ Hombres \% } & \multirow{2}{*}{ Sig. } \\
\hline & 1 & 2 & 3 & & & \\
\hline Es lo único que me permitirá salir adelante & $-0,785$ & & 0,302 & 63,3 & 68,2 & $*$ \\
\hline Aprendo cosas útiles que usaré después & & 0,547 & 0,324 & 22,3 & 23,0 & \\
\hline Hay otras cosas que son más importantes & 0,575 & 0,380 & & 5,4 & 6,4 & \\
\hline Para mí no es tan importante & 0,431 & $-0,494$ & $-0,470$ & 4,6 & 4,2 & \\
\hline Puedo tener buenos trabajos sin estudiar & 0,398 & & 0,720 & 6,8 & 8,2 & * \\
\hline Me servirá para ser profesional o técnico & 0,398 & 0,723 & & 43,2 & 33,9 & $* *$ \\
\hline Porcentaje de varianza explicada & 26,6 & 21,1 & 17,4 & & & \\
\hline
\end{tabular}

${ }^{*}$ Cargas de los componentes menores a 0,300 han sido sacadas para mayor claridad; $\mathrm{p}<0,05{ }^{* *} \mathrm{p}<0$

Fuente: elaboración propia. 
para permanecer en las escuelas regulares y finalizar su ciclo escolar. Tampoco hay un set de rasgos o patrones de vida que permita distinguir a los estudiantes que han repetido solo una vez de aquellos que tienen un historial de fracaso escolar. Parece ser que algunos estudiantes se alejaron del espacio escolar formal debido a que encontraron más satisfacción o "sentido" en otro tipo de actividades, comúnmente vinculadas a la interacción con otros, caracterizados por rechazar la ética escolar. Por otra parte, en el caso de las mujeres, ellas aparentemente han tenido dificultades en la escuela producto principalmente de problemas en su casa.

La diversidad de factores asociados al abandono escolar se desarrolla en los complejos e inestables entornos familiares y comunitarios en los que la mayoría de estos estudiantes han vivido sus vidas. El aprendizaje se ve facilitado por la regularidad y la consistencia; la incertidumbre sobre lo que ocurrirá en el futuro dificulta la adquisición de un repertorio de conductas eficaces. Las personas criadas en condiciones de gran incertidumbre son vistas por los demás como menos inteligentes y menos fiables. Como grupo, es más probable que se diferencien en mayor medida entre sí que un grupo de personas criadas en un contexto de mayor estabilidad. De este modo, aunque todos son desertores escolares, perciben el mundo, especialmente en relación con ellos mismos, de maneras diferentes. También difieren en su nivel de autocomprensión, en su capacidad para entender lo que más les gustaría. Los alumnos procedentes de hogares en los que existen buenas relaciones interpersonales y pocos problemas económicos podrían ser capaces de mencionar ambiciones u objetivos deseados. Al contrario, aquellos que provienen de hogares más turbulentos tienden a abarcar más, porque no están seguros de lo que quieren o de cuáles de sus objetivos pueden ser alcanzados (Espinoza et al., 2019b).

En suma, dada la heterogeneidad de los estudiantes y la variedad de factores que los llevaron a abandonar el sistema escolar formal, la capacidad de retener a estos jóvenes dependerá de la capacidad de los centros para proveer un programa que difiera del programa más uniforme que caracteriza a las escuelas regulares, uno que responda de la mejor manera posible a sus habilidades, intereses y expectativas (Leone y Drakeford, 1999). Esto exige, por ejemplo, proveer docentes comprometidos, con un currículum relevante, y motivar a los estudiantes para la consecución de sus sueños (Gilson, 2006; Sullivan, 2015). Ahora bien, a pesar de todo, prácticamente la mitad de los jóvenes de este estudio declaró no tener razones para abandonar sus actuales estudios, lo que muestra una respuesta favorable de estos estudiantes hacia los Centros de Segunda Oportunidad. Esto a su vez sugiere que el personal de estos establecimientos ha tenido un cierto éxito en la creación de un ambiente positivo y acogedor, citado por algunos autores como el determinante crítico de la persistencia en este tipo de escuelas (Blazar y Kraft, 2016; Ruzek et al., 2016).

\section{Conclusiones}

Los datos analizados revelan algunas importantes diferencias entre hombres y mujeres. En general, las mujeres mencionan más preferencias o expectativas a futuro, lo que implica que poseen una mayor preocupación respecto de cómo sustentarse en la vida adulta. Los hombres, por otro lado, se muestran relativamente menos preocupados acerca del empleo y las posibilidades educativas futuras. La asociación negativa entre relaciones en el hogar y estabilidad financiera familiar y el número de opciones futuras que los estudiantes mencionan es consistente con el supuesto de que la gente que está más preocupada o ansiosa acerca de su futuro considera más alternativas. Las mujeres consideran más opciones que los hombres con respecto a trabajo y dinero y educación, pero no acerca de dejar el hogar $u$ otros aspectos.

Por otra parte, algunos estudiantes se encuentran altamente motivados, en el sentido de desear tener éxito en el centro de segunda oportunidad; 
ahora bien, no existe una asociación clara entre su fracaso escolar en el sistema regular y su nivel de motivación actual. Además, pareciera no existir una relación entre expectativas laborales y educacionales en los estudiantes de los centros de segunda oportunidad, ya que se valora la educación en un nivel discursivo, lo que no se traduce en un deseo de ingresar a la universidad o de obtener un grado técnico o profesional. En cuanto a las variables relacionadas con las características de los estudiantes y de sus familias, son relativamente pocas las que están vinculadas de manera significativa con el fracaso escolar y la repitencia.
Por el propósito y el diseño de este estudio, los datos no dicen demasiado acerca del impacto de los centros de segunda oportunidad. Como consecuencia de ello, de este análisis no es posible extraer conclusiones sobre la mejor manera de organizar y operar escuelas de esta clase. No se cuenta aún con una caracterización de diversos tipos de estudiantes ni con un catálogo de intervenciones efectivas. Se espera entonces que estudios futuros hagan posible el poder distinguir entre los centros más y menos exitosos, e identificar aquellos elementos que en mayor medida contribuyen a la reescolarización de estos adolescentes y jóvenes.

\section{Referencias}

Adelman, M. y Székely, M. (2017). An overview of school dropout in Central America: Unresolved issues and new challenges for education progress. European Journal of Educational Research, 6(3), 235-259. DOI: https://doi. org/10.12973/eu-jer.6.3.235

Alarcón, A., Parella, S. y Yiu, J. (2014). Educational and occupational ambitions among the Spanish 'Second Generation': The case of Barcelona. Journal of Ethnic and Migration Studies, 40(10), 1614-1636. DOI: https://doi.or g/10.1080/1369183X.2013.831550

Alvarado, C. et al. (2014). La reintegración educativa: los colegios de segunda oportunidad como vía de restauración del derecho a la educación. Santiago de Chile: Corporación Opción.

Amitay, G. y Rahav, G. (2018). Attachment and pedagogical relevant practices as elements of a successful alternative school through the narratives of its students. Psychology in the Schools, 55(10), 1239-1258. DOI: https:// doi.org/10.1002/pits.22200

Antelm, A., Gil-López, A. y Cacheiro-González, M. (2015). Análisis del fracaso escolar desde la perspectiva del alumnado y su relación con el estilo de aprendizaje. Educación y Educadores, 18(3), 471-489. DOI: https://doi. org/10.5294/edu.2015.18.3.6

Bandura, A. (2006). Toward a psychology of human agency. Perspectives on Psychological Science, 1(2), 164-180. DOI: https://doi.org/10.1111/j.1745-6916.2006.00011.x

Behtoui, A. (2017). Social capital and the educational expectations of young people. European Educational Research Journal, 16(4), 487-503. DOI: https://doi.org/10.1177/1474904116682248 
Biblioteca del Congreso Nacional (2014). Factores asociados a la deserción escolar en Chile. Santiago de Chile: BCN. Recuperado de: https://www.bcn.cl/obtienearchivo?id=repositorio/10221/21112/5/Factores\%2Oasociados\%20a\%2ola\%2odesercion\%2oescolar\%2oen\%20Chile.pdf

Blazar, D. y Kraft, M. (2016). Teacher and teaching effects on student's attitudes and behaviors. Educational Evaluation and Policy Analysis, 39, 146-170. DOI: https://doi.org/10.3102/0162373716670260

Bohon, S. A., Kirkpatrick, M. y Gorman, B. K. (2006). College aspirations and expectations among Latino adolescents in the United States. Social Problems, 53(2), 207-225. DOI: https://doi.org/10.1525/sp.2006.53.2.207

Bowers, A. (2010). Grades and graduation: A longitudinal risk perspective to identify student dropouts. The Journal of Educational Research, 103(3), 191-207. DOI: https://doi.org/10.1080/00220670903382970

Bowers, A., Sprott, R. y Taff, S. (2013). Do we know who will drop out?: A review of the predictors of dropping out of high school: Precision, sensitivity, and specificity. The High School Journal, 96(2), 77-100. DOI: https://doi. org/10.1353/hsj.2013.0000

Bozick, R., Alexander, K., Entwisle, D., Dauber, S. y Kerr, K. (2010). Framing the future: Revisiting the place of educational expectations in status attainment. Social Forces, 88(5), 2027-2052. DOI: https://doi.org/10.1353/ sof.2010.0033

Bradley, C. y Renzulli, L. (2011). The complexity of non-completion: Being pushed or pulled to drop out of high school. Social Forces, 9o(2), 521-545. DOI: https://doi.org/10.1093/sf/soroo3

Brusilovsky, S. y Cabrera, M. E. (2005). Cultura escolar en educación media para adultos. Una tipología de sus orientaciones. Convergencia, 38, 277-311. Recuperado de: https://convergencia.uaemex.mx/article/view/1464

Brusilovsky, S., Cabrera, M. E. y Kloberdanz, C. (2010). Contención. Una función asignada a las escuelas para adultos. Revista Interamericana de Educación de Adultos, 32(1), 25-39. Recuperado de: https://www.redalyc. org/pdf/4575/457545094003.pdf

Castillo, D. (2003). Desertores de la educación básica: reflexiones e interrogantes a partir de la práctica. Revista Latinoamericana de Innovaciones Educativas, 37, 69-90.

CEAAL - Consejo de Educación Popular de América Latina y el Caribe (2013). A paso lento... Análisis de los avances del complimiento de la Confintea VI. Consejo de Educación Popular de América Latina y el Caribe. Lima: Consejo de Educación para Adultos de América Latina.

Ceja, M. (2004). Chicana college aspirations and the role of parents: Developing educational resiliency. Journal of Hispanic Higher Education, 3(4), 338-362. DOI: https://doi.org/10.1177/1538192704268428

CIAE - Centro de Investigación Avanzada en Educación (2019). Caracterización cuantitativa de trayectorias escolares en jóvenes con algún grado de exclusión educacional. Informe Técnico. Recuperado de: http://ciae. uchile.cl/download.php?file=noticias/oo_1552671019.pdf 
Crone, E. A. y Dahl, R. E. (2012). Understanding adolescence as a period of social-affective engagement and goal flexibility. Nature Reviews Neuroscience, 13(9), 636-650. DOI: https://doi.org/10.1038/nrn3313

Darling-Hammond, L. y Cook-Harvey, C. (2018). Educating the whole child: Improving school climate to support student success. Palo Alto: Learning Policy Institute.

D’Alessandre, V. y Mattioli, M. (2015). ¿Por qué los adolescentes dejan la escuela? Comentarios a los abordajes conceptuales sobre el abandono escolar en el nivel medio. Cuaderno Sistema de Información de Tendencias Educativas en América Latina, 21, 1-24. Recuperado de: http://repositorio.minedu.gob.pe/handle/123456789/5089

Day, L., Mozuraityte, N., Redgrave, K. y McCoshan, A. (2013). Preventing early school leaving in Europe-Lessons learned from second chance education. Bruselas: Comisión Europea. Recuperado de: http://ec.europa.eu/ dgs/education_culture/repository/education/library/study/2013/secondchance_en.pdf

De Witte, K. y Rogge, N (2013). Dropout from secondary education: All's well that begins well. European Journal of Education, 47(4), 1-20. DOI: https://doi.org/10.1111/ejed.12001

Donlan, A. E., Prescott, J. E. y Zaff, J. F. (2016). Differential predictors of academic achievement: Individual and familial factor interactions with community poverty. Journal of Children and Poverty, 22(2), 113-132. DOI: https://doi.org/10.1080/10796126.2016.1163665

Escobar, D., Berlien, K. y Ostoic, D. (2016). Informe final de evaluación: Programa Educación para Personas Jóvenes y Adultas (EPJA). Santiago: Dipres, Ministerio de Hacienda.

Espinoza, O., Castillo, D., González, L. E., Santa Cruz, E.y Loyola, J. (2014). Deserción escolar en Chile: un estudio de caso en relación a factores intraescolares. Educación y Educadores, 17(1), 32-50. DOI: https://doi.org/10.5294/ edu.2014.17.1.2

Espinoza, O., Loyola, J., Castillo, D. y González, L. E. (2016). Evaluación de los programas de reescolarización en Chile: La perspectiva de los estudiantes. Educação e Pesquisa, 42(4), 969-986. DOI: https://doi.org/10.159o/ s1517-9702201605142856

Espinoza, O., González, L. E., Castillo y Neut, S. (2018). Expectativas educacionales de estudiantes que concurren a escuelas de segunda oportunidad: la experiencia chilena. Revista Mexicana de Investigación Educativa, 23(79), 1171-1193. Recuperado de: http://www.scielo.org.mx/scielo.php?script=sci_arttext\&pid $=$ S1405-66662018000401171

Espinoza, O., González, L. E., Castillo y Neut, S. (2019a). Condicionantes de la retención estudiantil en escuelas de segunda oportunidad en Chile. Revista Latinoamericana de Ciencias Sociales, Niñez y Juventud, 17(2), 1-27. DOI: https://doi.org/10.11600/1692715x.17201

Espinoza, O., McGinn, N.y González, L. E. (2019b). Alternative education programs for high school age students in Chile. Education and Urban Society, 52(4), 561-589. DOI: https://doi.org/10.1177/0013124519879428 
Espinoza, O., González, L. E., McGinn, N. y Castillo, D. (2020a). What factors predict the engagement of dropouts in alternative schools in Chile? Improving Schools, 23(1), 43-67. DOI: https://doi.org/10.1177/1365480219864835

Espinoza, O., González, L. E., McGinn, N. y Castillo, D. (2020b). Engaging dropouts with differentiated practices: Some evidence from Chile. Research Papers in Education (marzo). DOI: https://doi.org/10.1080/02671522.20 20.1736615

Estrada, M. J. (2015). Las jóvenes que desertan de la educación media: virajes y puntos de no retorno. Revista Latinoamericana de Ciencias Sociales, Niñez y Juventud, 13(2), 995-1008. DOI: https://doi.org/10.11600/1692 $715 \times .13231190214$

Fernández-Suárez, A., Herrero, J., Pérez, B., Juarros-Basterretxea, J. y Rodríguez-Díaz, F. (2016). Risk factors for school dropout in a sample of juvenile offenders. Frontiers in Psychology, 26. DOI: https://doi.org/10.3389/ fpsyg.2016.01993

Foley, K., Gallipoli, G.y Green, D (2014). Ability, parental valuation of education and high school dropout decision. Journal of Human Resources, 49(4), 906-944. DOI: https://doi.org/10.1353/jhr.2014.0027

Gilson, T. (2006). Alternative high schools: What types of programs lead to the greatest level of effectiveness? Normes, 6(1), 48-66. https://files.eric.ed.gov/fulltext/EJ844648.pdf

Glewwe, P., Hanushek, E. A., Humpage, S. y Ravina, R. (2011). School resources and educational outcomes in developing countries: A review of the literature from 1990 to 2010. National Bureau of Economic Research Working Paper, 17554. DOI: https://doi.org/10.3386/W17554

González, L. E., Espinoza, Ó. y López, L. (2013). Deserción y fracaso académico en la educación superior en América Latina y el Caribe: resultados e implicancias. En B. Steren, J. Arriaga y M. Costa (coords.), Una visión integral del abandono (pp. 21-60). Porto Alegre: Edipucrs.

Gutman, L. M., Schoon, I. y Sabates, R. (2012). Uncertain aspirations for continuing in education: Antecedents and associated outcomes. Developmental Psychology, 48, 1707-1718. DOI: https://doi.org/10.1037/a0026547

Hawkins, R., Jaccard, J. y Needle, E. (2013). Non-academic factors associated with dropping out of high school: Adolescent problem behaviors. Journal of the Society for Social Work and Research, 4(2), 58-75. DOI: https:// doi.org/10.5243/jsswr.2013.5

Hennig, C., Pineda-Báez, C. y Vargas, A. (2019). School engagement for avoiding dropout in middle school education. International Education Studies, 12(5), 35-48. DOI: https://doi.org/10.5539/ies.v12n5P35

Ingrum, A. (2007). High school dropout determinants: The effect of poverty and learning disabilities. The Park Place Economist, 14, 73-79. Recuperado de: https://digitalcommons.iwu.edu/parkplace/vol14/iss1/16/

Jacob, B. A. y Wilder, T. (2010). Educational expectations and attainment. National Bureau of Economic Research Working Paper, 15683. DOI: https://doi.org/10.3386/w15683 
Laghi, F., Liga, F., Baumgartner, E. y Baiocco, R. (2012). The perspective and psychosocial positive functioning among Italian adolescents who binge eat and drink. Adolescence, 35(5), 1277-1284. DOI: https://doi. org/10.1016/j.adolescence.2012.04.014

Lamb, S. y Markussen, E. (2011). School dropout and completion: An international perspective. En S. Lamb, R. Teese, J. Polesel y N. Sandberg (eds.), School dropout and completion: International comparative studies in theory and policy (pp. 1-18). Holanda: Springer.

León, J. y Sugimaru, C. (2017). Las expectativas educativas de los estudiantes de secundaria de regiones amazónicas: un análisis de los factores asociados desde el enfoque de eficacia escolar. Documentos de Investigación, Grupo de Análisis para el Desarrollo, 85. Recuperado de: https://pdfs.semanticscholar.org/baec/ e76a1azoecc6bc7cd6ca3f5da271add17eg2.pdf

Leone, P.y Drakeford, W. (1999). Alternative education: From a "last chance" to a proactive model. The Clearing House: A Journal of EducationalStrategies, Issues and Ideas, 72(3), 86-88. DOI: https://doi.org/10.1080/00098659909600154

López de Mesa-Melo, C., Carvajal-Castillo, C., Urrea-Roa, P. y Soto-Godoy, M. (2013). Factores asociados a la convivencia escolar en adolescentes. Educación y Educadores, 16(3), 383-410. DOI: https://doi.org/10.5294/ edu.2013.16.3.1

Maxwell, S., McNeely, C. y Carboni, J. (2016). Cultural capital efficacy in parental and student aligned expectations for postsecondary matriculation. Journal of Education and Human Development, 5(1), 1-12. DOI: https://doi.org/10.15640/jehd.v5n1a1

McDermott, E., Anderson, S. y Zaff, J. (2018). Dropout typologies: Relating profiles of risk and support to later educational re-engagement. Applied Developmental Science, 22, 217-232. DOI: https://doi.org/10.1080/1088 8691.2016.1270764

Mills, M. y McGregor, G. (2014). Re-engaging young people in education: Learning from alternative schools. Londres: Routledge.

MIDE-UC/Servicio Nacional de Menores - Sename (2016). Estudio de caracterización de los programas de reinserción educativa de Sename y Mineduc. Recuperado de: http://www.sename.cl/web/wp-content/ uploads/2016/10/Estudio_Caract_Prog_Reins_Sename-Mineduc.pdf

MINEDUC (2010). Diagnóstico, análisis y recomendaciones para el rediseño e instalación en régimen de la línea nivelación de estudios modalidad flexible del Programa Chilecalifica. Santiago de Chile: Ministerio de Educación.

MINEDUC (2015). Resultados adjudicación de proyectos de reinserción 2015. Santiago de Chile: Ministerio de Educación.

Noltemeyer, A., Ward, R. y Mcloughlin, C. (2015). Relationship between school suspension and student outcomes: A meta-analysis. School Psychology Review, 44(2), 224-240. 
OECD - Organisation for Economic Co-operation and Development (2017). Students' expectations of further education. En PISA 2015 Results (Volume III): Students' Well-Being (pp. 103-113). París: OECD. DOI: https://doi. org/10.1787/9789264273856-10-en

OECD - Organisation for Economic Co-operation and Development (2018). Education at a glance 2018. Bruselas: OECD.

Osorio, J. (2013). Desafíos docentes en la educación de jóvenes y adultos en Chile: hacia una agenda de conversaciones para el diseño e implementación de nuevas políticas. Temas de Educación, 19, 57-65. Recuperado de: https://revistas.userena.cl/index.php/teduacion/article/view/380

Palomar, J. y Victorio, A. (2016). Expectativas laborales en la adolescencia: correlatos psicosociales. Interdisciplinaria, 33(1), 95-110. DOI: https://doi.org/10.16888/interd.2016.33.1.6

Palomar, J. y Victorio, A. (2017). Expectativas educativas de adolescentes mexicanos en condiciones de pobreza. Revista de Psicología, 26(1), 1-11. DOI: https://doi.org/10.5354/0719-0581.2017.46393

Parker, P., Jerrim, J., Schoon, I. y Marsh, H. (2016). A multination study of socioeconomic inequality in expectations for progression to higher education: The role of between-school tracking and ability stratification. American Educational Research Journal, 53(1), 6-32. DOI: https://doi.org/10.3102/0002831215621786

Peña, J., Soto, V. y Calderón, U. (2016). La influencia de la familia en la deserción escolar: estudio de caso en estudiantes de secundaria de dos instituciones de las comunas de Padre las Casas y Villarrica, Región de la Araucanía, Chile. Revista Mexicana de Investigación Educativa, 21(70), 881-899. Recuperado de: http://www. scielo.org.mx/scielo.php?script=sci_arttext\&pid=S1405-66662016000300881

Peña, M. y Toledo, C. (2017). Ser pobre en el Chile neoliberal: estudio discursivo en una escuela vulnerable. Revista Latinoamericana de Ciencias Sociales, Niñez y Juventud, 15(1), 207-218. Recuperado de: http://www.scielo. org.co/scielo.php?script=sci_arttext\&pid=S1692-715X2017000100013

Perreira, K., Harris, K. y Lee, D. (2006). Making it in America: High school completion by immigrant and native youth. Demography, 43(3), 511-536. DOI: https://doi.org/10.1353/dem.2006.0026

Pizarro, M., De la Vega, L. y Ormazábal, M. (2016). Evaluación programas gubernamentales (EPG). Programa reinserción escolar. Santiago de Chile: Dirección de Presupuestos, Ministerio de Hacienda.

Porowski, A., O'Conner, R. y Luo, J. (2014). How do states define alternative education? Key findings. Washington: Department of Education, Institute of Education Sciences, National Center for Education Evaluation and Regional Assistance, Regional Educational Laboratory Mid-Atlantic.

Ramírez, A. (2020). Alternativas a la segregación escolar: estrategias desde la educación no formal. Educación y Educadores, 23(4), 637-656. DOI: https://doi.org/10.5294/edu.2020.23.4.5

Reddick, J., Welton, A. D., Alsandor, J. D., Denyszyn, J. L. y Platt, C. S. (2011). Stories of success: High minority, high poverty public school graduate narratives on accessing higher education. Journal of Advanced Academics, 22(4), 594-618. DOI: http://doi.org/10.1177/1932202X11414133 
Román, M. (2013). Factores asociados al abandono y la deserción escolar en América Latina: una mirada en conjunto. Reice, Revista Iberoamericana sobre Calidad, Eficacia y Cambio en Educación, 11(2), 33-59. Recuperado de: https://www.redalyc.org/pdf/551/55127024002.pdf

Romo, J. y Cumsille, P. (2020). Caracterización de estudiantes con trayectorias educativas interrumpidas en una muestra de escuelas de reingreso a través de análisis de clases latentes. Pensamiento Educativo, 57(1), 1-21. DOI: https://doi.org/10.7764/PEL.57.1.2020.4

Rumberger, R. W.y Lim, S. A. (2008). Why students drop out of school: A review of 25 years of research. Los Ángeles: Dropout Research Project.

Rumberger, R.W. (2011). Dropping out. Boston: Harvard University Press.

Ruzek, E., Hafen, C., Allen, J., Gregory, A., Mikami, A. y Pianta, R. (2016). How teacher emotional support motivates students: The mediating roles of perceived peer relatedness, autonomy support, and competence. Learning and Instruction, 42, 95-103. DOI: https://doi.org/10.1016/j.learninstruc.2016.01.004

Sabates, R., Akyeampong, K., Westbrook, J. y Hunt, F. (2010). School drop out: Patterns, causes, changes and policies. [Documento de programa 2011/ED/EFA/MRT/PI/O8] Unesco. Recuperado de: https://unesdoc.unesco. org/ark:/48223/pfoooo190771

Salinas-Quiroz, F., Silva, P., Cambón, V. y Fraga, S. (2017). Asistencia intermitente y deserción en Educación Inicial. Testimonios de madres uruguayas. Revista Latinoamericana de Ciencias Sociales, Niñez y Juventud, 15(2), 913-925. Recuperado de: http://revistaumanizales.cinde.org.co/rlcsnj/index.php/Revista-Latinoamericana/article/view/2472/

Salva, F., Nadal, J. y Melià-Barceló, M. A. (2016). Itinerarios de éxito y rupturas en la educación de segunda oportunidad. Revista Latinoamericana de Ciencias Sociales, Niñez y Juventud, 14(2), 1405-1419. Recuperado de: http://revistaumanizales.cinde.org.co/rlcsnj/index.php/Revista-Latinoamericana/article/view/2615

Schuller, T. y Watson, D. (2009). Learning through life. Londres: Niace.

Seginer, R. (2009). Future orientation. Developmental and ecological perspectives. Israel: Springer.

Stinebrickner, R. y Stinebrickner, T. R. (2013). Academic performance and college dropout: Using longitudinal expectations data to estimate a learning model. [CIBC Working Papers] Centre for Human Capital and Productivity, University of Western Ontario, Londres.

Stewart, E. B., Stewart, E. A. y Simons, R. L. (2007). The effect of neighborhood context on the college aspiration of African American adolescents. American Education Research Journal, 44(4), 896-919. DOI: https://doi. org/10.3102/0002831207308637

Sullivan, T. (2015). The difference between more effective and less effective alternative schools: A study of alternative schools in the Greater Los Angeles Area. Long Beach: California State University. 
ISSN 0123-1294 | e-ISSN 2027-5358 | Educ.Educ. Vol. 24. No.1 | Febrero-abril de 2021 | pp. 113-134

Universidad de La Sabana | Facultad de Educación

Tedesco, J. C. (1998). Desafios de las reformas educativas en América Latina. Buenos Aires: IIPE.

Tynkkynen, L., Vuori, J. y Salmela-Aro, K. (2012). The role of psychological control, socioeconomic status and academic achievement in parents' educational aspirations for their adolescent children. European Journal of Developmental Psychology, 9, 695-710. DOI: https://doi.org/10.1080/17405629.2012.671581

Unesco Institute for Statistics (2012). Opportunities lost: The impact of grade repetition and early school leaving. Montreal: Unesco.

Vallee, D. (2017). Student engagement and inclusive education: Reframing student engagement. International Journal of Inclusive Education, 21(9), 920-937. DOI: https://doi.org/10.1080/13603116.2017.1296033

Verdugo, L. (2017). Estudio sobre el nivel de ajuste académico y las expectativas de futuro de los adolescentes. Publicaciones Didácticas, 82, 424-431. Recuperado de: https://pdfs.semanticscholar.org/1coe/9e453296207c 9ccoad7c52ca958a437d7fd2.pdf

Vivanco, S. (2014). La reintegración educativa. Los colegios de segunda oportunidad como vía de restauración del derecho a la educación. Santiago de Chile: Universidad Central. 